\section{Immunohistochemical localization of periostin in human gingiva}

\author{
T. Cobo, ${ }^{1,2}$ A. Obaya, ${ }^{3}$ S. Cal, $, 4,5$ \\ L. Solares, ${ }^{4}$ R. Cabo, ${ }^{6}$ J.A. Vega, ${ }^{6,7}$ \\ J. Cobo ${ }^{1,2}$ \\ 'Instituto Asturiano de Odontología, \\ Oviedo, Spain \\ 2Departamento de Cirugía y \\ Especialidades Médico-Quirúrgicas, \\ Universidad de Oviedo, Spain \\ ${ }^{3}$ Departamento de Biología Funcional, \\ Área de Fisiología, Universidad de \\ Oviedo, Spain \\ ${ }^{4}$ Departamento de Bioquímica y Biología \\ Molecular, Universidad de Oviedo, Spain \\ ${ }^{5}$ Instituto Universitario de Oncología del \\ Principado de Asturias, Oviedo, Spain \\ 'Departamento de Morfología y Biología \\ Celular, Grupo SINPOS, Universidad de \\ Oviedo, Spain \\ ${ }^{7}$ Facultad de Ciencias de la Salud, \\ Universidad Autónoma de Chile, \\ Santiago, Chile
}

\section{Abstract}

The periostin is a matricellular protein expressed in collagen-rich tissues including some dental and periodontal tissues where it is regulated by mechanical forces, growth factors and cytokines. Interestingly the expression of this protein has been found modified in different gingival pathologies although the expression of periostin in normal human gingiva was never investigated. Here we used Western blot and double immunofluorescence coupled to laser-confocal microscopy to investigated the occurrence and distribution of periostin in different segments of the human gingival in healthy subjects. By Western blot a protein band with an estimated molecular mass of $94 \mathrm{kDa}$ was observed. Periostin was localized at the epithelial-connective tissue junction, or among the fibers of the periodontal ligament, and never co-localized with cytokeratin or vimentin thus suggesting it is an extracellular protein. These results demonstrate the occurrence of periostin in adult human gingiva; its localization suggests a role in the bidirectional interactions between the connective tissue and the epithelial cells, and therefore in the physiopathological conditions in which these interactions are altered.

\section{Introduction}

The matricellular proteins are a heterogeneous group of extracellular matrix (ECM) proteins that interacts with either other ECM proteins and with cell-surface receptors, growth factors and cytokines., ${ }^{1,2}$ The periostin, also called osteoblast-specific factor 2 (OSF$2),{ }^{3}$ is a non-structural matricellular protein that directly interacts with type I collagen and fibronectin regulating the biomechanical properties of connective tissues. ${ }^{4}$ It is an 811 amino acid, $93 \mathrm{kDa}$ secreted ECM protein and four main isoforms have been identified which are not uniformly but differentially expressed in various cell lines; ${ }^{3,5}$ a human periodontal ligament specific isoform has been recently characterized. ${ }^{6}$

Periostin is present in collagen-rich connective tissues like bone, skeletal muscle, tendons, joint ligaments, periodontal ligament, heart valves, adipose tissue, or skin. ${ }^{7}$ At present the functions of periostin in several biomedical areas like osteology, oncology, cardiovascular and respiratory systems, inflammatory diseases or dentistry, in both normal and pathological conditions, are rather well known. As a whole its main functions seem to be during the development and tissue repair, because the predominant role in mesenchymal remodeling. ${ }^{8}$ A series of recent studies have detected expression of periostin in developing and mature dental tissues, ${ }^{9}$ alveolar bone or periodontal ligament. ${ }^{5,10-12}$ Consistently, mice deficient in periostin display changes in ECM of dental tissues ${ }^{13,14}$ as well as defects in mineralized tissues..$^{15,16}$ By contrast little information is available about the occurrence and distribution of periostin in the gingiva. It is known that gingival fibroblasts may be a source of periostin in response to some cytokines which are elevated in periodontitis, ${ }^{17}$ and decreased levels of periostin were found in gingival crevicular fluid proportionally with the progression and severity of periodontal disease. ${ }^{18}$ Moreover, periostin is involved in signaling pathways of drug-induced gingival overgrowth. ${ }^{19}$

Since no data are available about the distribution of periostin in adult human gingival in normal conditions, and because its potential role in gingival pathologies, we used Western blot and immunohistochemistry to analyze the occurrence and localization of periostin in these tissues. To establish whether periostin is cellular or extracellular we labeled in parallel keratinocytes and dermal fibroblasts. This study may serve as a baseline for future researches in gingival pathologies.
Correspondence: Dr. José A. Vega, Departamento de Morfología y Biología Celular, Facultad de Medicina, Universidad de Oviedo, Avd. Julián Clavería 6, 33006 Oviedo, Spain.

Tel. +34.985.104097; Fax +34.985.1013618

E-mail: javega@uniovi.es

Key words: Periostin; matricellular proteins; human gingiva.

Acknowledgements: this study was supported by Instituto Asturiano de Odontologia, Oviedo, Spain.

Received for publication: 28 June 2015. Accepted for publication: 22 August 2015.

This work is licensed under a Creative Commons Attribution NonCommercial 3.0 License (CC BYNC 3.0).

(C) Copyright T. Cobo et al., 2015

Licensee PAGEPress, Italy

European Journal of Histochemistry 2015; 59:2548 doi:10.4081/ejh.2015.2548

\section{Materials and Methods}

\section{Materials}

Samples of gingival tissues (sized 2x2x2 $\mathrm{mm}$, approximately) were surgically excised from healthy patients undergoing orthodontic treatment from zones not exposed to orthodontic forces, and were obtained from the Instituto Asturiano de Odontologia. The age range was 16 to 32 years, and were males $(n=8)$ and females $(n=4)$. The tissue samples were washed with tap water followed with cold saline, then fixed in buffered $10 \%$ formalin for $24 \mathrm{~h}$, and routinely processed for paraffin embedding. The pieces were cut $10 \mu \mathrm{m}$ thick and the sections mounted on gelatine-coated microscope slides. Moreover, fresh samples ( $n=6$ from different subject) were quickly frozen, stored at $-80^{\circ} \mathrm{C}$ and used for Western blot. This study was approved by the Ethics Committee of Instituto Asturiano de Odontologia (Oviedo, Spain) and informed consent was obtained from each subject.

\section{Western blot analysis}

Lysates prepared from gingival homogenates were processed as follows: representative samples of the free gingival, attached gingival and non-bone attached periodontal ligament were pooled and homogenized $(1: 2, \mathrm{w} / \mathrm{v})$ in Tris-HCl buffered saline (TBS, $0.1 \mathrm{M}$, pH 7.5) containing $1 \mathrm{M}$ leupeptin, $10 \mathrm{M}$ pepstatin and $2 \mathrm{mM}$ phenylmethylsulfonyl fluoride. The homogenates were centrifuged at $25,000 \mathrm{rpm}$ for $15 \mathrm{~min}$ at $4^{\circ} \mathrm{C}$ and the resulting pellet dissolved in Tris $\mathrm{HCl} 10 \mathrm{mM} \mathrm{pH} \mathrm{6.8,2 \%} \mathrm{SDS,} 100$ $\mathrm{mM}$ dithiothreitol, and $10 \%$ glycerol at $4^{\circ} \mathrm{C}$. 
The lysates were analyzed by electrophoresis in $12 \%$ discontinuous polyacrylamide SDS gels. After electrophoresis, proteins were transferred to a nitrocellulose membrane and antibody non-specific binding was blocked by immersion for $3 \mathrm{~h}$ in PBS containing 5\% dry milk, and $0.1 \%$ Tween-20. The membranes were then incubated at $4^{\circ} \mathrm{C}$ for $2 \mathrm{~h}$ with a rabbit polyclonal antibody against a peptide from fasciclin domain 1 of mouse periostin (LSBL10443, LifeSpan BioSciences, Inc., Seattle, WA, USA), used diluted 1:200. After incubation, the membranes were washed with TBS pH 7.6 containing 20\% Tween-20, and incubated again for $1 \mathrm{~h}$ with goat anti-rabbit IgG (diluted 1:100) at room temperature. Membranes were washed again and incubated with the PAP complex diluted 1:100 for $1 \mathrm{~h}$ at room temperature. Finally, the reaction was developed using a chemiluminescent reagent (ECL, Amersham Pharmacia Biotech, Buckinghamshire, UK) and exposed to Hyperfilm. Marker proteins were visualized by staining with Brilliant Blue. Relative values of periostin were estimated by imageJ quantification in the three different segments of the gingiva. $\beta$-actin was used as loading control.

\section{Double immunofluorescence}

Deparaffinized and rehydrated sections were processed for immunohistochemical detection of periostin using the EnVision Antibody Complex Kit (Dako, Copenhagen, Denmark) following the manufacturer's recommendations. The anti-periostin antibody was the same described above. Moreover, antivimentin (clone 334, Boehringer-Mannheim, Mannheim, Germany), anti-pancytokeratin (clone PCK-26, Sigma-Aldrich Quimica, SL, Madrid, Spain) mouse monoclonal antibodies, and anti-collagen type I rabbit monoclonal antibody (ERP778, Abcam, Cambridge, UK) were used to label dermal fibroblasts, epithelial cells and type I collagen fibrils, respectively. The sections were processed for simultaneous detection of periostin and vimentin, and periostin and pan-cytokeratin, as follows: the non-specific binding was reduced by incubation for 30 min with a solution of $1 \%$ bovine serum albumin in TBS. The sections were then incubated overnight, at $4^{\circ} \mathrm{C}$ in a humid chamber with a 1:1 mixture of anti-periostin and anti-vimentin antibodies (both diluted 1:100 in the blocking solution); or anti-periostin and anti-pancytokeratin antibodies (diluted 1:200 and 1:100, respectively, in the blocking solution); or anti-vimentin and anti-type I collagen (both diluted 1:100 in the blocking solution). After rinsing with TBS, the sections were incubated for 1 hour with Alexa fluor 488-conjugated goat anti-rabbit IgG (Serotec, Oxford, UK), diluted 1:1000 in TBS containing 5\% mouse serum (Serotec), then rinsed again, and incu- bated for another hour with $\mathrm{Cy}^{\mathrm{TM}} 3$-conjugated donkey anti-mouse antibody (JacksonImmunoResearch, Baltimore, MD, USA) diluted 1:50 in TBS. Both steps were performed at room temperature in a dark humid chamber. Finally, to ascertain structural details sections were counterstained and mounted with DAPI diluted in glycerol medium $(10 \mathrm{ng} / \mathrm{mL})$. Triple fluorescence was detected using a Leica DMRXA automatic fluorescence microscope (Photonic Microscopy Service, University of Oviedo) coupled with a Leica Confocal Software, ver. 2.5 (Leica Microsystems $\mathrm{GmbH}$, Heidelberg, Germany) and the images captured were processed using the software Image $\mathrm{J}$ version $1.43 \mathrm{~g}$, Master Biophotonics Facility, Mac Master University Ontario (www.macbiophotonics.ca). For control purposes representative sections were processed in the same way as described above using nonimmune rabbit or mouse sera instead of the primary antibodies, or omitting the primary antibodies in the incubation. Under these conditions no specific immunostaining was observed.

\section{Results}

The expression of periostin at the protein level in the human gingiva was analyzed using Western blot and immunohistochemistry. In gingival homogenates Western blot associated to the anti-periostin antibody used throughout this study detected a single protein band with an estimated molecular weight of $94 \mathrm{kDa}$ (Figure 1a) which is consistent with than expected for the human periostin. Image J densitometric analysis demonstrated that the higher relative levels of periostin with respect to $\beta$-actin corresponded to the non-bone attached periodontal ligament segment, followed by free gingiva and attached gingiva (Figure 1b).

To map the histological distribution of periostin in the human gingiva we used double immunofluorescence coupled with laser-confocal microscopy in three different sectors: the free gingiva, the attached gingiva, and the segment of connective tissue related to the junctional epithelium (which is especially rich in

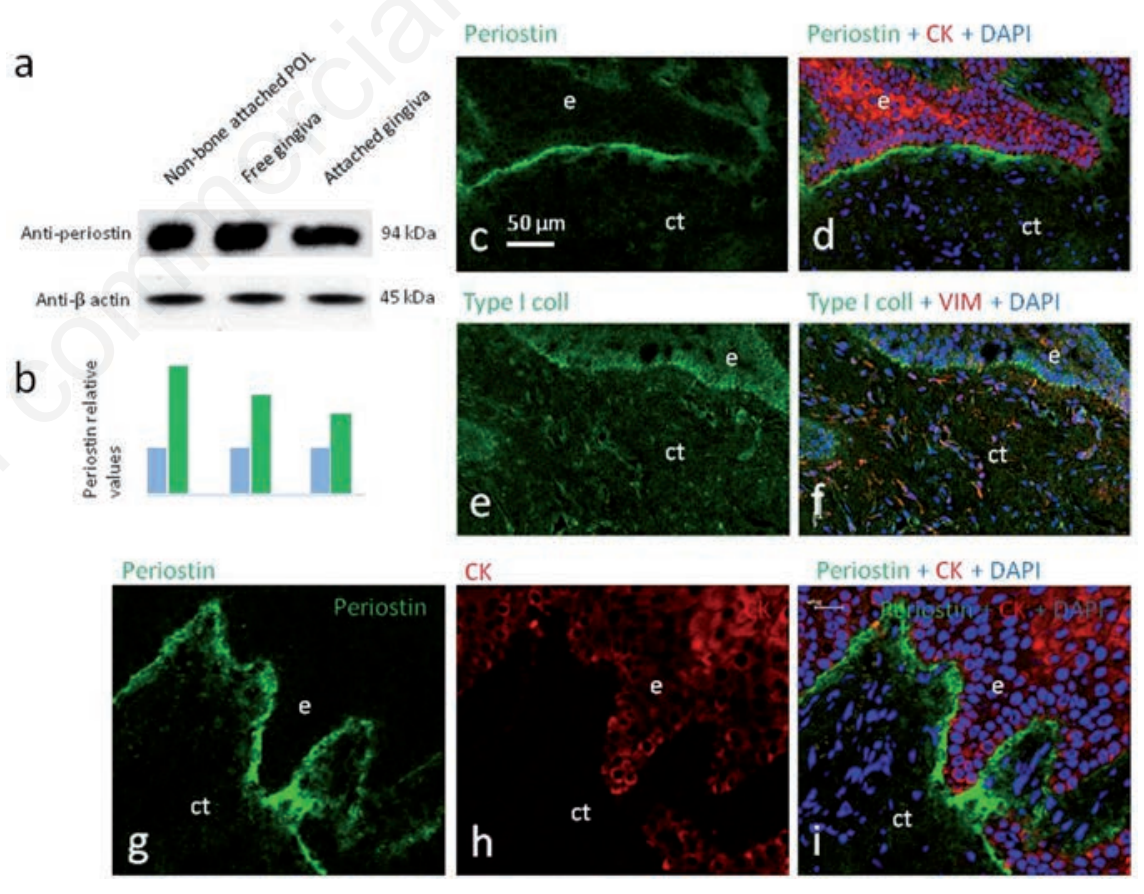

Figure 1. Western blot and immunohistochemical detection of periostin in adult human gingiva. The anti-periostin antibody used recognizes a single protein band of about $94 \mathrm{kDa}$ consistent with that expected for periostin (a). Image J analysis demonstrating relative levels of periostin with respect to $\beta$-actin in the different segments considered (b). Confocal laserscanning images of periostin (c,d,g,i; green,), cytokeratin (d,h,i; red,), vimentin (f; red,), and type $I$ collagen (e, green) in adult human gingiva. Specific immunoreactivity for periostin was restricted to the epithelial-connective tissue junction. No co-localization of periostin with cytokeratin or vimentin was observed. Periostin has a similar localization of type I collagen. c-f) Objective 40x/1.25 Oil; pinhole airy 1 , XY resolution $156 \mathrm{~nm}$ and Z resolution $334 \mathrm{~nm}$. g-i) Objective 63x/1.40 Oil; pinhole airy 1.55, XY resolution $139 \mathrm{~nm}$ and $Z$ resolution $232 \mathrm{~nm}$. ct, connective tissue; e, epithelium. 
fibers of the periodontal ligament). The results were compared with the pattern of localization of cytokeratin and vimentin. As a rule periostin was never localized within the cells but always in the extracellular space.

In the sections processed for the simultaneous demonstration of periostin plus cytokeratin (Figure $1 \mathrm{c}, \mathrm{d}, \mathrm{g}, \mathrm{h}$ ) it was observed that periostin-immunoreactivity is concentrated at the epithelium-connective tissue junction, presumably associated to ECM proteins of the basal membrane and was never found within the cytoplasm of the basal epithelial cells. On the other hand, the distribution of the immunoreactivity for periostin and type I collagen were similar at the epithelium-connective tissue junction (Figure $1 \mathrm{e}, \mathrm{f}$ ) although a faint widespread immunostaining for type I collagen was also detected in the connective tissue. Double immunofluorescence was also carried out for periostin and vimentin in order to determine the relation of periostin with the connective tissue fibroblasts. Independently of the gingival segment analyzed, free (Figure 2 a-c) or attached gingival (Figure 2 d-f), periostin immunoreactivity was dissociated from the vimentin positive fibroblast and was restricted to the epithelial-connective tissue junction. Regarding to the gingival segment containing the non-bone attached periodontal ligament, disposed under the junctional epithelium, the distribution of the periostin immunoreactivity was irregular, and never colocalized with the vimentin-positive fibroblasts (Figure 2 g-i). Although some images occasionally might suggest co-localization of periositin witn vimentin or cytokeratin 2D cytofluorograms from the two detection channels of periostin (green) and cytokeratin or vimentin (red) demonstrated absence of colocalization (Figure $2 \mathrm{j}-\mathrm{l}$ ).

As a summary, in human normal gingiva periostin is extracellular and is restricted to the epithelial-connective tissue junction, and among the fibroblasts forming the non-bone attached segments of the periodontal ligament.

\section{Discussion}

This study was designed to investigate the occurrence and distribution of the segregated matricellular protein periostin in the human gingiva. The localization of periostin in the dental tissues and the periodontal ligament in now rather well known, ${ }^{8}$ but no information is available about its distribution in the gingiva in spite of its potential role in the pathophysiology of some gingival disorders. ${ }^{17-19}$

The protein we have identified by Western blot has a molecular weight of $94 \mathrm{kDa}$. This is in good agreement with the expected molecular mass of this protein $(93.3 \mathrm{kDa}),{ }^{3}$ and was similar to that found in other human epithelial tissues like the cornea. ${ }^{20}$ The small differences between our results and others could be related to occurrence of tissue specific periostin isoforms, or post-translational processes of the protein. Based on image densitometry the relative level of periostin varies among different segments of the gingiva. The reason for these differences might be related to the density of cells producing periostin, and the functional significance of these findings if any remain to be elucidated.

We have regularly observed that periostin in normal gingiva is restricted to the epitheliumconnective tissue junction, and is also present around the fibers of the non-bone attached portion of the periodontal ligament. This pattern of distribution was independent of the gingival segment considered. As far as we know this is the first study reporting the distribution of periostin in the human gingiva. In agreement with the identification of periostin as a matricellular protein we observed that it is localized extracellularly. Nevertheless, other studies on epithelial tissues reported the presence of periostin immunoreactivity in the basal keratinocytes, basal lamina, and dermal fibroblasts in healthy human skin, ${ }^{21,22}$ or in the basal layer cells of the human corneal epithelium..$^{20}$ According to those authors periostin only becomes extracellular during tissue remodeling of wound repair. ${ }^{21,22}$ Since in normal gingiva periostin is exclusively extracellular, its function may be distinct from that of skin, ${ }^{17}$ and the presence of intracellular periostin probably reflects non-secreted protein.

The role of periostin in the interactions of the connective tissue and the epithelial cells has been extensively studied, ${ }^{8}$ however it remains still unknown in the gingiva. In the skin periostin plays pivotal functions in collagen fibrillogenesis, collagen cross-linking, and the formation of ECM meshwork via interactions with other ECM components. Whether or not it is the same for the gingiva remains to be investigated. In the skin periostin expression is enhanced by mechanical stress or skin injury. This is indicative of the physiologically protec-

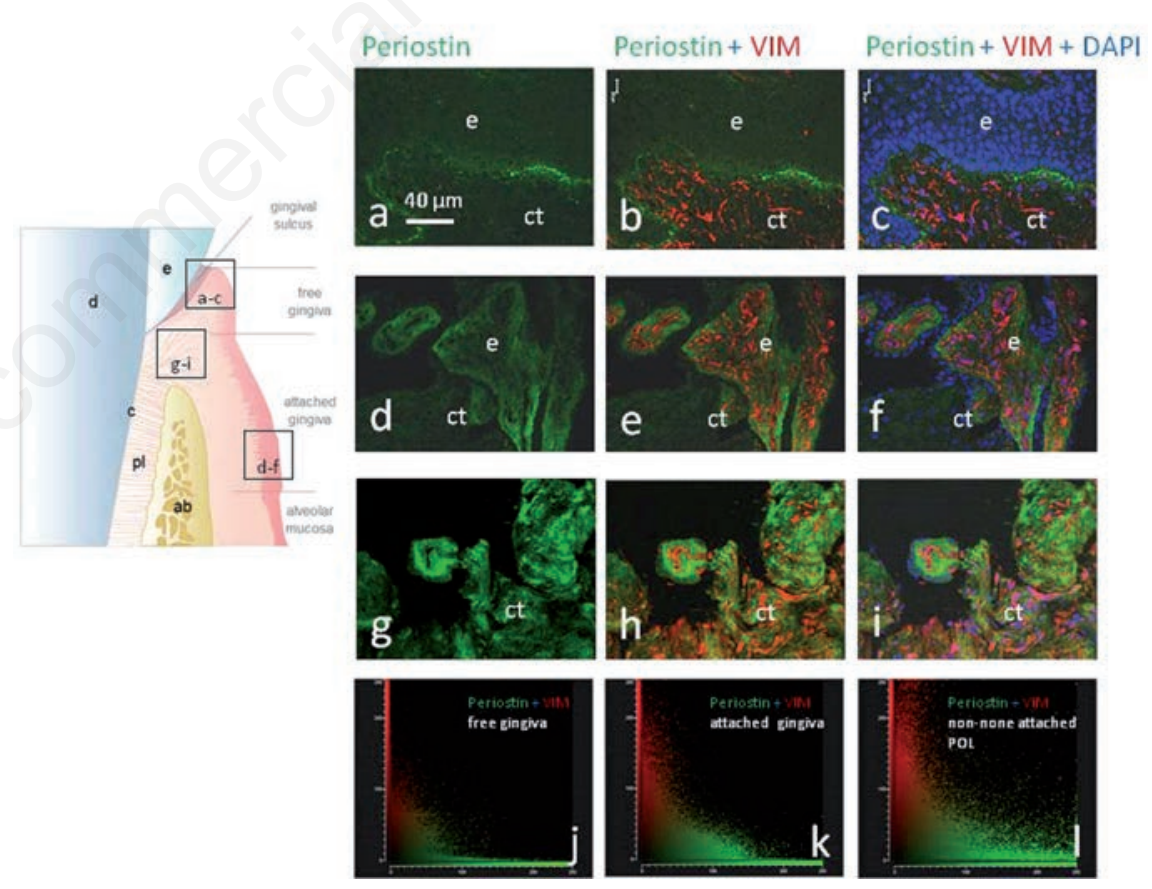

Figure 2. Schematic representation of a section of the human gingiva showing the different segments of the epithelium: free gingival (a-c), attached gingival (d-f) and the subjunctional epithelium gingival. Panels a to $i$ are confocal laser-scanning images of periostin (green) and vimentin (red) in the free (a-c), attached (d-f) and sub-junctional epithelium (g-i) of adult human gingiva. Periostin immunoreactivity was never co-localized with vimentin in the fibroblast of the connective tissue or the periodontal ligament, but formed a layer of variable thickness at the epithelial-connective tissue junction or among the fibres of the periodontal ligament. Objective 40x/1.25 Oil; pinhole airy 1, XY resolution $156 \mathrm{~nm}$ and $\mathrm{Z}$ resolution $334 \mathrm{~nm}$. Panels $\mathrm{j}$, $\mathrm{k}$ and 1 show a $2 \mathrm{D}$ cytofluorogram from the two detection channels from the original image showing no co-localization of the assessed antigens. e, epithelium; ct, connective tissue. 
tive functions of periostin, which promotes wound repair ${ }^{23}$ producing myofibroblast differentiation, keratinocytes proliferation and fibroblast proliferation and migration. ${ }^{24}$ The localization of periostin in the gingiva and the since it is an ECM secreted protein suggest it could signal in both epithelial cells. Matricellular proteins appear to be of importance in collagen assembly, ${ }^{25}$ and the expression of periostin in the epithelial-connective tissue junction suggests it may influence the biology of the basal membrane, including collagen fibrillogenesis (especially type I collagen which colocalizes) but probably its presence may be also related to cell migration, proliferation and adhesion of fibroblasts. ${ }^{26}$ On the other hand, the free and sub-junctional epithelium segments of the gingival contain fibers of the periodontal ligament subjected to mechanical stress which in turn may activate latent TGF- 1 and increase periostin mRNA in periodontal ligament fibroblasts. ${ }^{12}$ Therefore, periostin may participate in the maintenance of the structure and reparative processes in adult human gingival as it occurs in the skin, although in these tissues periostin have different localization, extracellular $v s$ intracellular. ${ }^{21,22}$

As a summary, our study has demonstrated the localization of periostin in the ECM of the periodontal ligament and the cell-free zone of the dental pulp in adult human teeth. These results might serve as a baseline for future studies in pathological conditions.

\section{References}

1. Bornstein P, Sage EH. Matricellular proteins: extracellular modulators of cell function. Curr Opin Cell Biol 2002;14:608-16.

2. Roberts DD. Emerging functions of matricellular proteins. Cell Mol Life Sci 2011;68:3133-6.

3. Takeshita S, Kikuno R, Tezuka K, Amann E. Osteoblast-specific factor 2: cloning of a putative bone adhesion protein with homology with the insect protein fasciclin I. Biochem J 1993;294:271-88.

4. Norris RA, Damon B, Mironov V, Kasyanov V, Ramamurthi A, Moreno-Rodriguez R, et al. Periostin regulates collagen fibrillogenesis and the biomechanical properties of connective tissues. J Cell Biochem 2007;101 695-711.

5. Horiuchi K, Amizuka N, Takeshita S, Takamatsu H, Katsuura M, Ozawa H, et al.
Identification and characterization of a novel protein, periostin, with restricted expression to periosteum and periodontal ligament and increased expression by transforming growth factor beta. J Bone Miner Res 1999;14:1239-49.

6. Yamada S, Tauchi T, Awata T, Maeda K, Kajikawa T, Yanagita M. et al. Characterization of a novel periodontal ligament-specific periostin isoform. J Dent Res 2014;93:891-7.

7. Kudo A. Periostin in fibrillogenesis for tissue regeneration: periostin actions inside and outside the cell. Cell Mol Life Sci 2011;68:3201-7.

8. Conway SJ, Izuhara K, Kudo Y, Litvin J, Markwald R, Ouyang G, et al. The role of periostin in tissue remodeling across health and disease. Cell Mol Life Sci 2014;71:1279-88.

9. Wiesen RM, Padial-Molina M, Volk SL, McDonald N, Chiego D Jr, Botero T, et al. The expression of periostin in dental pulp cells. Arch Oral Biol 2015;60:760-7.

10. Wilde J, Yokozeki M, Terai K, Kudo A, Moriyama K. The divergent expression of periostin mRNA in the periodontal ligament during experimental tooth movement. Cell Tissue Res 2003;312:345-51.

11. Suzuki H, Amizuka N, Kii I, Kawano Y, Nozawa-Inoue K, Suzuki A, et al. Immunohistochemical localization of periostin in tooth and its surrounding tissues in mouse mandibles during development. Anat Rec A Discov Mol Cell Evol Biol 2004;281:1264-75.

12. Rios HF, Ma D, Xie Y, Giannobile WV, Bonewald LF, Conway SJ, et al. Periostin is essential for the integrity and function of the periodontal ligament during occlusal loading in mice. J Periodontol 2008;79: 1480-90.

13. Lv S, Liu H, Cui J, Hasegawa T, Hongo H, Feng W, et al. Histochemical examination of cathepsin K, MMP1 and MMP2 in compressed periodontal ligament during orthodontic tooth movement in periostin deficient mice. J Mol Histol 2014;45:303-9.

14. Tabata C, Hongo H, Sasaki M, Hasegawa T, de Freitas PH, Yamada T, et al. Altered distribution of extracellular matrix proteins in the periodontal ligament of periostindeficient mice. Histol Histopathol 2014;29:731-42.

15. Rios H, Koushik SS, Wang H, Zhou HM, Lindsley A, Rogers R, et al. Periostin null mice exhibit dwarfism, incisor enamel defects, and an early-onset periodontal disease-like phenotype. Mol Cell Biol 2005; 25:11131-44.

16. Ma D, Zhang R, Sun Y, Rios HF, Haruyama $\mathrm{N}, \mathrm{Han} \mathrm{X}$, et al. A novel role of periostin in postnatal tooth formation and mineralization. J Biol Chem 2011;286:4302-9.

17. Nakajima M, Honda T, Miyauchi S, Yamazaki K. Th2 cytokines efficiently stimulate periostin production in gingival fibroblasts but periostin does not induce an inflammatory response in gingival epithelial cells. Arch Oral Biol 2014;59:93-101.

18. Balli U, Keles ZP, Avci B, Guler S, Cetinkaya B0, Keles GC. Assessment of periostin levels in serum and gingival crevicular fluid of patients with periodontal disease. J Periodontal Res doi: 10.1111/jre.12254. [Epub ahead of print]

19. Trackman PC, Kantarci A. Molecular and clinical aspects of drug-induced gingival overgrowth. J Dent Res 94: 540-6.

20. Qu Y, Chi W, Hua X, Deng R, Li J, Liu Z, Pflugfelder SC, et al. Unique expression pattern and functional role of periostin in human limbal stem cells. PLoS One 2015;10:e0117139.

21. Jackson-Boeters L, Wen W, Hamilton DW. Periostin localizes to cells in normal skin, but is associated with the extracellular matrix during wound repair. J Cell Commun Signal 2009;3:125-33.

22. Ontsuka K, Kotobuki Y, Shiraishi H, Serada S, Ohta S, Tanemura A, et al. Periostin, a matricellular protein, accelerates cutaneous wound repair by activating dermal fibroblasts. Exp Dermatol 2012;21: 331-6.

23. Yamaguchi Y. Periostin in skin tissue and skin-related diseases. Allergol Int 63:161-70.

24. Elliott CG, Kim SS, Hamilton DW. Functional significance of periostin in excisional skin repair: is the devil in the detail? Cell Adh Migr 2012;6:319-26.

25. Bornstein P, Agah A, Kyriakides TR. The role of thrombospondins 1 and 2 in the regulation of cell-matrix interactions, collagen fibril formation, and the response to injury. Int J Biochem Cell Biol 2004;36: 1115-25.

26. Padial-Molina M, Volk SL, Rios HF. Periostin increases migration and proliferation of human periodontal ligament fibroblasts challenged by tumor necrosis factor $-\alpha$ and Porphyromonas gingivalis lipopolysaccharides. J Periodontal Res 2014;49:405-14. 